\title{
Holomorphic Equivariant Cohomology via a Transversal Holomorphic Vector Field*
}

\author{
Huitao Feng
}

\begin{abstract}
In this paper an analytic proof of a generalization of a theorem of Bismut ([Bis1, Theorem 5.1]) is given, which says that, when $v$ is a transversal holomorphic vector field on a compact complex manifold $X$ with a zero point set $Y$, the embedding $j: Y \rightarrow X$ induces a natural isomorphism between the holomorphic equivariant cohomology of $X$ via $v$ with coefficients in $\xi$ and the Dolbeault cohomology of $Y$ with coefficients in $\left.\xi\right|_{Y}$, where $\xi \rightarrow X$ is a holomorphic vector bundle over $X$.
\end{abstract}

2000MR Subject Classification 53C, 58J

\section{Introduction}

For a compact complex manifold $X$, let $T_{\mathbf{C}} X$ denote the complexification of the real tangent bundle $T_{\mathbf{R}} X$ of $X$. Then $T_{\mathbf{C}} X$ splits canonically as $T_{\mathbf{C}} X=T X \oplus \overline{T X}$, where $T X$ and $\overline{T X}$ are the holomorphic and the anti-holomorphic tangent bundle of $X$, respectively. Let $\xi \rightarrow X$ be a holomorphic vector bundle over $X$. There exists a natural $\mathbf{Z}$-grading in $\Lambda^{\prime}\left(T_{\mathbf{C}}^{*} X\right) \otimes \xi$ defined by the following decomposition:

$$
\Lambda^{\prime}\left(T_{\mathbf{C}}^{*} X\right) \otimes \xi=\bigoplus_{-\operatorname{dim} X \leq r \leq \operatorname{dim} X} \Lambda^{(r)}\left(T_{\mathbf{C}}^{*} X\right) \otimes \xi
$$

where

$$
\Lambda^{(r)}\left(T_{\mathbf{C}}^{*} X\right) \otimes \xi=\bigoplus_{q-p=r} \Lambda^{q}\left(\overline{T^{*} X}\right) \otimes\left(\Lambda^{p}\left(T^{*} X\right) \otimes \xi\right) .
$$

We will use $\Lambda^{(\cdot)}\left(T_{\mathbf{C}}^{*} X\right) \otimes \xi$ to denote $\Lambda^{\cdot}\left(T_{\mathbf{C}}^{*} X\right) \otimes \xi$ with this Z Z grading. Let $\Omega^{(r)}(X, \xi)$ (resp. $\Omega^{(\cdot)}(X, \xi)$ ) be the complex vector space of smooth sections of $\Lambda^{(r)}\left(T_{\mathbf{C}}^{*} X\right) \otimes \xi$ (resp.

*Partially supported by the NNSF of China (10271059) and the K. C. Wong Education Foundation 
$\left.\Lambda^{(\cdot)}\left(T_{\mathbf{C}}^{*} X\right) \otimes \xi\right)$. Then $\left(\Omega^{(\cdot)}(X, \xi)=\bigoplus_{r} \Omega^{(r)}(X, \xi), \bar{\partial}^{X}\right)$ is a complex and the cohomology groups $H^{(r)}(X, \xi)$ associated to this complex are direct sums:

$$
H^{(r)}(X, \xi)=\bigoplus_{q-p=r} H^{p, q}(X, \xi),
$$

where $H^{p, q}(X, \xi)$ are the usual Dolbeault cohomology groups of $X$ with coefficients in the holomorphic vector bundle $\xi$.

For any holomorphic vector field $v$ on $X$, set

$$
\bar{\partial}_{v}^{X}=\bar{\partial}^{X}+i(v): \Omega^{(\cdot)}(X, \xi) \rightarrow \Omega^{(\cdot+1)}(X, \xi),
$$

where $i(v)$ is the standard contraction operator defined by $v$. The consideration of operators $\bar{\partial}^{X}$ and $i(v)$ together goes back to [Bot]. Clearly, $\left(\Omega^{(\cdot)}(X, \xi), \bar{\partial}_{v}^{X}\right)$ is also a $\mathbf{Z}$-graded complex. Denote the cohomology groups associated to this complex by $H_{v}^{(r)}(X, \xi)$, which are called the holomorphic equivariant cohomology groups of $X$ via $v$ with coefficients in $\xi$ (cf. [L]).

In [CL], Carrell and Lieberman discussed the relation between Dolbeault cohomology of a connected compact Kähler manifold $X$ and the zero point set $Y$ of a holomorphic vector field $v$ on $X$ by using Deligne degeneracy criterion and proved that $H^{(r)}(X, \mathbf{C})$ vanished for all $|r|>\operatorname{dim} Y$ if $Y \neq \emptyset$. Since $\operatorname{dim} H_{v}^{(\cdot)}(X, \mathbf{C})=\operatorname{dim} H^{(\cdot)}(X, \mathbf{C})$ in this case (see [L, Theorem 1.3] and [CL]), the corresponding vanishing results for $H_{v}^{(\cdot)}(X, \mathbf{C})$ are also valid. For a general compact complex manifold $X$ and a transversal holomorphic vector field $v$ on $X$ (see a definition in [Bis1, Sect.5.1]), Liu in [L] proved that $\operatorname{dim} H^{(\cdot)}(Y, \mathbf{C}) \leq$ $\operatorname{dim} H_{v}^{(\cdot)}(X, \mathbf{C})$ by constructing an injective homomorphism $\alpha_{r}: H^{(r)}(Y, \mathbf{C}) \rightarrow H_{v}^{(\cdot)}(X, \mathbf{C})$. Moreover, Liu got a counting formula for $\operatorname{dim} H_{v}^{(0)}(X, \mathbf{C})$ in term of the multiplicities of the zero points of $v$ if the zero points of $v$ are discrete. Under the assumption that the zero point of $v$ is nodegenerate, motivated by Witten's deformation idea ([W]), he also sketched an analytic proof of his formula in [L, Sect.7] by examining the behavior of a natural deformation $D_{T}^{X}$ of the Riemann-Roch operator on $X$ as $T \rightarrow \infty$.

A more general result in this direction is due to Bismut. By using the technique of spectral sequences in [Bis1, Theorem 5.1], Bismut proves that, when $v$ is a transversal holomorphic vector field on $X$ with a zero point set $Y$, the embedding $j: Y \rightarrow X$ induces naturally a quasi-isomorphism $j^{*}:\left(\Omega^{(\cdot)}(X, \mathbf{C}), \bar{\partial}_{v}^{X}\right) \rightarrow\left(\Omega^{(\cdot)}(Y, \mathbf{C}), \bar{\partial}^{Y}\right)$.

In this paper, we will give an analytic proof of the following fairly straightforward generalization of the Bismut's theorem [Bis1, Theorem 5.1]: 
Theorem 1.1 Let $v$ be a transversal holomorphic vector field on a compact complex manifold $X$ with a zero point set $Y$. Then

$$
j^{*}:\left(\Omega^{(\cdot)}(X, \xi), \bar{\partial}_{v}^{X}\right) \rightarrow\left(\Omega^{(\cdot)}\left(Y,\left.\xi\right|_{Y}\right), \bar{\partial}^{Y}\right)
$$

is a quasi-isomorphism.

Following Witten's deformation idea ([W]) as in [L, Sect.7], we will also work with a deformation $D_{T}^{X}$ of the twisted Riemann-Roch operator on $X$ by $\xi$ but the whole proof now is heavily based on the analytic localization techniques developed by Bismut and Lebeau (cf. [BL]), since the analysis involved here is much complicated than the situation in $\left[\mathrm{L}\right.$, Sect.7]. A key point in our proof is to express $D_{T}^{X}$ and to trivialize the bundle on which $D_{T}^{X}$ acts by using the Bismut connection $\nabla^{-B}$ (see [Bis2, II. b)]). We should point out that if the trivialization is made by a lifting of the holomorphic Hermitian connection $\nabla^{T X}$ on $T X$, then an extra term coming from the torsion of $\nabla^{T X}$ will enter to the final operator on $Y$ in the Bismut-Lebeau localization process of $D_{T}^{X}$ as $T \rightarrow \infty$. But it is not clear to us that the extra term is zero for a general complex manifold $X$. Consequently, we could only obtain an equality related to the involved operators at the index level.

\section{A deformed twisted Riemann-Roch operator $D_{T}^{X}$ and its local behavior near $Y$}

This section is divided into three parts. In a) we introduce a deformation $D_{T}^{X}$ of the twisted Riemann-Roch operator by $\xi$ via $v$, which has been used in [L, Sect.7] in the case of $\xi=\mathbf{C}$. In b) we recall the definition of the Bismut connection $\nabla^{-B}$ (cf. [Bis2, II. b)]) and express $D_{T}^{X}$ in this connection by a direct application of [Bis2, Theorem 2.2]. In c) we study the local behavior of the deformed operator $D_{T}^{X}$ near the submanifold $Y$ following [BL, Sect.8], in which the Bismut connection will play an essential role.

\section{a) A deformed twisted Riemann-Roch operator $D_{T}^{X}$}

Let $X$ be a compact complex manifold of $\mathbf{C}$-dimension $n$. For any $T \in \mathbf{R}$, we consider the following deformed operator

$$
\bar{\partial}_{T}^{X}=\bar{\partial}+T i(v): \Omega^{(\cdot)}(X, \xi) \rightarrow \Omega^{(\cdot+1)}(X, \xi)
$$


and the deformed complex $\left(\Omega^{(\cdot)}(X, \xi), \bar{\partial}_{T}^{X}\right)$. One verifies easily as the proof of $[\mathrm{L}$, Lemma 1.1] that the cohomologies associated to this deformed complex do not depend on $T \neq 0$.

Let $g^{T X}$ (resp. $g^{\xi}$ ) be a Hermitian metric on $X$ (resp. $\xi$ ). By the standard procedure there is an induced Hermitian metric $\langle,\rangle_{\Lambda^{(\cdot)}\left(T_{\mathbf{C}}^{*} X\right) \otimes \xi}$ on $\Lambda^{(\cdot)}\left(T_{\mathbf{C}}^{*} X\right) \otimes \xi$. Let $d v_{X}$ denote the Riemannian volume element of $\left(X, g^{T X}\right)$. Then for $s_{1}, s_{2} \in \Omega^{(\cdot)}(X, \xi)$,

$$
\left\langle\left\langle s_{1}, s_{2}\right\rangle\right\rangle=\frac{1}{(2 \pi)^{\operatorname{dim}_{\mathbf{C}} X}} \int_{X}\left\langle s_{1}, s_{2}\right\rangle_{\Lambda^{(\cdot)}\left(T_{\mathbf{C}}^{*} X\right) \otimes \xi} d v_{X}
$$

defines an $L^{2}$-Hermitian inner product on $\Omega^{(\cdot)}(X, \xi)$. Let $\bar{\partial}^{X *}$ and $\bar{\partial}_{T}^{X *}$ denote the formal adjoint operators of $\bar{\partial}^{X}$ and $\bar{\partial}_{T}^{X}$, respectively. For any $U \in T_{\mathbf{C}} X$, let $U^{*}$ be an element in $T_{\mathbf{C}}^{*} X$ defined by $g^{T X}(U, \cdot)$. Clearly, $\bar{v}^{*}$ is a $(1,0)$-form on $X$ and $\bar{v}^{*} \wedge$ is the dual operator of $i(v)$. Moreover, we have

$$
\bar{\partial}_{T}^{X *}=\bar{\partial}^{X *}+T \bar{v}^{*} \wedge: \Omega^{(\cdot)}(X, \xi) \rightarrow \Omega^{(\cdot-1)}(X, \xi) .
$$

Set

$$
D^{X}=\sqrt{2}\left(\bar{\partial}^{X}+\bar{\partial}^{X *}\right), \quad D_{T}^{X}=\sqrt{2}\left(\bar{\partial}_{T}^{X}+\bar{\partial}_{T}^{X *}\right) .
$$

Clearly, $D^{X}$ is the usual twisted Riemann-Roch operator by the holomorphic bundle $\xi$ and $D_{T}^{X}$ is a deformation of $D^{X}$ and interchanges $\Omega^{(\text {even })}(X, \xi)$ and $\Omega^{(\text {odd })}(X, \xi)$. From Hodge theory we have the following isomorphisms

$$
\left.\operatorname{ker}\left(D_{T}^{X}\right)^{2}\right|_{\Omega^{(r)}(X, \xi)} \cong H_{v}^{(r)}(X, \xi) .
$$

Lemma 2.1 For any open neighborhood $\mathcal{U}$ of $Y$, there exist constants $a>0, b>0$ and $T_{0}>0$ such that for any $s \in \Omega^{(\cdot)}(X, \xi)$ with Supp $s \subset X \backslash \mathcal{U}$ and any $T \geq T_{0}$, one has the following estimate for Sobolev norms,

$$
\left\|D_{T}^{X} s\right\|_{0}^{2} \geq a\left(\|s\|_{1}^{2}+(T-b)\|s\|_{0}^{2}\right)
$$

Proof. An easy computation shows that

$$
D_{T}^{X, 2}=D^{X, 2}+2 T^{2}|v|^{2}+2 T\left(\left(\bar{\partial}^{X} \bar{v}^{*}\right) \wedge+i\left(\bar{\partial}^{X} \bar{v}^{*}\right)\right)
$$

where $i\left(\bar{\partial}^{X} \bar{v}^{*}\right)$ denotes the adjoint operator of $\left(\bar{\partial}^{X} \bar{v}^{*}\right) \wedge$. Note that $\left(\left(\bar{\partial}^{X} \bar{v}^{*}\right) \wedge+i\left(\bar{\partial}^{X} \bar{v}^{*}\right)\right)$ is a zero order operator and $v \neq 0$ on $X \backslash \mathcal{U}$, the lemma follows from the well-known Garding's inequality directly. 
By Lemma 2.1 and Hodge theory, we can study $H_{v}^{(\cdot)}(X, \xi)$ through the behavior of the operator $D_{T}^{X}$ near $Y$ for large $T$. Also by Lemma 2.1, it is an easy observation that, when $Y=\emptyset$, the cohomology group $H_{v}^{(\cdot)}(X, \xi)$ vanishes. In the following we will always assume that $v$ is transversal and $Y \neq \emptyset$. Note that generally $Y$ consists of some connected components $Y_{k}$ with different $\mathbf{C}$-dimensions $l_{k}$. When no confusion arises, we always drop the subscripts and simply denote them by $Y$ and $l$, respectively.

\section{b) An expression of $D_{T}^{X}$ in the Bismut connection}

We first recall the definition of the Bismut connection in [Bis2, II. b)]. For a complex manifold $X$ with a Hermitian metric $g^{T X}$, let $\nabla^{T X}$ be the holomorphic Hermitian connection on $T X$. Note that $\nabla^{T X}$ induces naturally an Euclidean connection on $T_{\mathbf{R}} X$ which preserves the complex structure of $T_{\mathbf{R}} X$. Let $T_{X}$ denote the torsion tensor of the connection $\nabla^{T X}$. Let $B_{X}$ be the antisymmetrization of the tensor $(U, V, W) \rightarrow \frac{1}{4}\left\langle T_{X}(U, V), W\right\rangle$ and let $S^{-B_{X}}$ denote the one form with values in antisymmetric elements of $\operatorname{End}\left(T_{\mathbf{R}} X\right)$ which is such that

$$
\left\langle S^{-B_{X}}(U) V, W\right\rangle=-2 B_{X}(U, V, W),
$$

where $U, V, W \in T_{\mathbf{R}} X$. Let $\nabla^{L^{X}}$ be the Levi-Civita connection on $T_{\mathbf{R}} X$. Set

$$
S_{X}=\nabla^{T X}-\nabla^{L^{X}} .
$$

Then $S_{X}$ is also a one form with values in antisymmetric elements of $\operatorname{End}\left(T_{\mathbf{R}} X\right)$. The important thing here is that $S^{-B_{X}}-S_{X}$ preserves the complex structure of $T_{\mathbf{R}} X$ (cf. [Bis2, (2.38)]). Now the Bismut connection $\nabla^{-B_{X}}$ on $T_{\mathbf{R}} X$ is defined by (cf. [Bis2, (2.37)])

$$
\nabla^{-B_{X}}=\nabla^{T X}+\left(S^{-B_{X}}-S_{X}\right)=\nabla^{L_{X}}+S^{-B_{X}} .
$$

The Bismut connection $\nabla^{-B_{X}}$ also preserves the complex structure of $T_{\mathbf{R}} X$ and so induces naturally a unitary connection on $T X$ and a unitary connection on $\overline{T X}$, which are still denoted by $\nabla^{-B_{X}}$. Note that when $X$ is Kähler, the Bismut connection $\nabla^{-B_{X}}$ coincides with the holomorphic Hermitian connection $\nabla^{T X}$. There is a unitary connection on $\Lambda^{\prime}\left(\overline{T^{*} X}\right)$ lifted canonically from $\nabla^{-B_{X}}$, which we still call the Bismut connection and denote by the same notation $\nabla^{-B_{X}}$.

Let $Y$ be a complex submanifold of $X$. Let $\pi: N \rightarrow Y$ be the normal bundle of $Y$ in $X$. We identify $N$ with the sub-bundle of $\left.T X\right|_{Y}$ orthogonal to $T Y$ with respect to the restriction metric $g^{\left.T X\right|_{Y}}$ on $\left.T X\right|_{Y}$ by $g^{T X}$. So we have the identification of $C^{\infty}$ bundles $\left.T X\right|_{Y}=T Y \oplus N$. Let $g^{T Y}$ (resp. $g^{N}$ ) be the induced metric on $T Y$ (resp. $N$ ) from $g^{\left.T X\right|_{Y}}$. Let $P^{T_{\mathbf{R}} Y}, P^{N_{\mathbf{R}}}$ be the orthogonal projection operators from $\left.T_{\mathbf{R}} X\right|_{Y}$ onto $T_{\mathbf{R}} Y$ and $N_{\mathbf{R}}$ 
respectively. Let $j$ denote the embedding of $Y$ into $X$. Then $j^{*} \nabla^{-B_{X}}$ is a connection on $\left.T_{\mathbf{R}} X\right|_{Y}$ preserving the metric $g^{\left.T X\right|_{Y}}$ and the complex structure of $\left.T_{\mathbf{R}} X\right|_{Y}$. Moreover, $P^{T_{\mathbf{R}} Y}\left(j^{*} \nabla^{-B_{X}}\right) P^{T_{\mathbf{R}} Y}$ is exactly the Bismut connection on $T_{\mathbf{R}} Y$ associated to the induce metric $g^{T Y}$, and $P^{N_{\mathbf{R}}}\left(j^{*} \nabla^{-B_{X}}\right) P^{N_{\mathbf{R}}}$ is a connection on $N_{\mathbf{R}}$ preserving the metric $g^{N}$ and the complex structure of $N_{\mathbf{R}}$. Set

$$
\begin{gathered}
\nabla^{-B_{X}, \oplus}=\nabla^{-B_{Y}} \oplus\left(P^{N_{\mathbf{R}}}\left(j^{*} \nabla^{-B_{X}}\right) P^{N_{\mathbf{R}}}\right), \\
\mathbf{A}=j^{*} \nabla^{-B_{X}}-\nabla^{-B_{X}, \oplus} .
\end{gathered}
$$

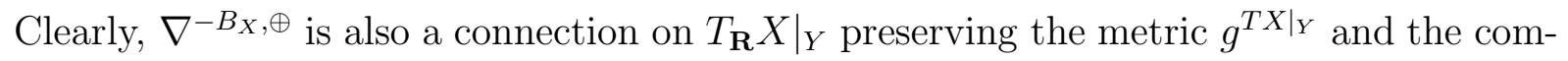
plex structure of $\left.T_{\mathbf{R}} X\right|_{Y}$, and $\mathbf{A}$ is the second fundamental form of the Bismut connection $\nabla^{-B_{X}}$. We still use the same notation $\nabla^{-B_{X}, \oplus}$ to denote its restriction on $\left.\overline{T X}\right|_{Y}$ as well as its lifting on $\Lambda \cdot\left(\left.\overline{T^{*} X}\right|_{Y}\right)$.

Now we return to our situation and express the deformed twisted Riemann-Roch operator $D_{T}^{X}$ in the Bismut connection by applying [Bis2, Theorem 2.2]. To do this we still need a holomorphic Hermitian connection on the bundle $\Lambda^{*}\left(T^{*} X\right) \otimes \xi$. Since our problem does not depend on the metrics, we can and will choose a special metric on the holomorphic bundle $\Lambda\left(T^{*} X\right)$ to simplify the analysis.

Let $\mathbf{L}_{v}:\left.\left.T X\right|_{Y} \rightarrow T X\right|_{Y}$ be the holomorphic Lie homomorphism defined by $\mathbf{L}_{v}(u)=$ $[v, u]$ for any $\left.u \in T X\right|_{Y}$. Denote $\mathbf{L}_{v}\left(\left.T X\right|_{Y}\right)$ by $\widetilde{N}$. Since $v$ is transversal, $\left.T X\right|_{Y}$ splits holomorphically into $T Y \oplus \widetilde{N}$ and $\mathbf{L}_{v}$ induces an isomorphism from $N$ to $\widetilde{N}$, which we still denote by $\mathbf{L}_{v}$. We introduce a new Hermitian metric $\widetilde{g}^{\widetilde{N}}$ on $\widetilde{N}$ by requiring that $\mathbf{L}_{v}: N \rightarrow$ $\widetilde{N}$ is unitary fiberwisely. Consequently, we get a new Hermitian metric $\tilde{g}^{\left.T X\right|_{Y}}=g^{T Y} \oplus \tilde{g}^{\widetilde{N}}$ on $\left.T X\right|_{Y}$. We can and we will extend $\tilde{g}^{\left.T X\right|_{Y}}$ to a Hermitian metric $\tilde{g}^{T X}$ on $T X$. We will denote $T X$ (resp. $\left.T X\right|_{Y}$ ) with the metric $\tilde{g}^{T X}$ (resp. $\tilde{g}^{\left.T X\right|_{Y}}$ ) by $\widetilde{T X}\left(\operatorname{resp} .\left.\widetilde{T X}\right|_{Y}\right.$ ) to distinguish the same bundle with different metrics. Let $\nabla^{\widetilde{T X}}$ (resp. $\left.\nabla^{\widetilde{T X}}\right|_{Y}$, resp. $\nabla^{\widetilde{N}}$ ) be the holomorphic Hermitian connection on $\widetilde{T X}$ (resp. $\left.\widetilde{T X}\right|_{Y}$, resp. $\left.\widetilde{N}\right)$. We have the following standard fact:

$$
j^{*} \nabla^{\widetilde{T X}}=\nabla^{\left.\widetilde{T X}\right|_{Y}}=\nabla^{T Y} \oplus \nabla^{\widetilde{N}} .
$$

We lift the holomorphic Hermitian connection $\nabla^{\widetilde{T X}}\left(\right.$ resp. $\nabla^{T Y}$ ) to the holomorphic Hermitian connection $\nabla^{\Lambda^{*}\left(\widetilde{T^{*} X}\right)}\left(\operatorname{resp} . \nabla^{\Lambda^{*}\left(T^{*} Y\right)}\right)$ on $\Lambda^{\cdot}\left(\widetilde{T^{*} X}\right)\left(\operatorname{resp} . \Lambda^{*}\left(T^{*} Y\right)\right)$.

Let $g^{\xi}$ be a Hermitian metric on $\xi$ and let $\nabla^{\xi}$ be the holomorphic Hermitian connection on $\xi$. Set $\left.\nabla^{\xi}\right|_{Y}=j^{*} \nabla^{\xi}$, which is the holomorphic Hermitian connection on $\left.\xi\right|_{Y}$. So $\nabla^{\Lambda^{\cdot}\left(\widetilde{T^{*} X}\right) \otimes \xi}=\nabla^{\Lambda^{\cdot}\left(\widetilde{T^{*} X}\right)} \otimes 1+1 \otimes \nabla^{\xi}$ (resp. $\left.\nabla^{\left.\Lambda^{\cdot}\left(T^{*} Y\right) \otimes \xi\right|_{Y}}=\nabla^{\Lambda^{*}\left(T^{*} Y\right)} \otimes 1+1 \otimes \nabla^{\left.\xi\right|_{Y}}\right)$ is a 
holomorphic Hermitian connection on $\Lambda^{\cdot}\left(\widetilde{T^{*} X}\right) \otimes \xi\left(\operatorname{resp} .\left.\Lambda^{\cdot}\left(T^{*} Y\right) \otimes \xi\right|_{Y}\right)$. Therefore,

$$
\begin{aligned}
& \nabla^{B, X}=\nabla^{-B_{X}} \otimes 1+1 \otimes \nabla^{\Lambda^{\cdot}\left(\widetilde{T^{*} X}\right) \otimes \xi}, \\
& \nabla^{B, Y}=\nabla^{-B_{Y}} \otimes 1+1 \otimes \nabla^{\left.\Lambda^{\cdot}\left(T^{*} Y\right) \otimes \xi\right|_{Y}}
\end{aligned}
$$

are unitary connections on the Hermitian vector bundle $\Lambda^{(\cdot)}\left(T_{\mathbf{C}}^{*} X\right) \otimes \xi,\left.\Lambda^{(\cdot)}\left(T_{\mathbf{C}}^{*} Y\right) \otimes \xi\right|_{Y}$ respectively.

For $U \in\left(T X, g^{T X}\right)$, set

$$
c(U)=\sqrt{2} U^{*} \wedge, \quad c(\bar{U})=-\sqrt{2} i(\bar{U})
$$

and for $U \in\left(\widetilde{T X}, \tilde{g}^{T X}\right)$, set

$$
\hat{c}(U)=-\sqrt{-2} i(U), \quad \hat{c}(\bar{U})=-\sqrt{-2} \bar{U}^{*} \wedge .
$$

We extend the map $c($ resp. $\hat{c})$ by $\mathbf{C}$ linearity into the Clifford action of $T_{\mathbf{C}} X$ (resp. $\widetilde{T_{\mathbf{C}} X}$ ) on $\Lambda \cdot\left(\overline{T^{*} X}\right)\left(\operatorname{resp} . \Lambda \cdot\left(\widetilde{T^{*} X}\right)\right)$.

Let $\left\{e_{1}, \ldots, e_{2 n}\right\}$ be an orthonormal basis $T_{\mathbf{R}} X$. Set

$$
c\left(B_{X}\right)=\frac{1}{6} \sum_{i, j, k=1}^{2 n} B_{X}\left(e_{i}, e_{j}, e_{k}\right) c\left(e_{i}\right) c\left(e_{j}\right) c\left(e_{k}\right) .
$$

Note that

$$
\frac{1}{4} \sum_{i, j, k=1}^{2 n}\left\langle S^{-B_{X}}\left(e_{i}\right) e_{j}, e_{k}\right\rangle c\left(e_{i}\right) c\left(e_{j}\right) c\left(e_{k}\right)=-3 c\left(B_{X}\right) .
$$

Now recall the definition (2.10) and apply [Bis2, Theorem 2.2] directly, we obtain the following expressions of $D^{Y}$ and $D_{T}^{X}$ with respect to the orthonormal basis $\left\{e_{1}^{\prime}, \ldots, e_{2 l}^{\prime}\right\}$ for $T_{\mathbf{R}} Y$ and $\left\{e_{1}, \ldots, e_{2 n}\right\}$ for $T_{\mathbf{R}} X$, respectively:

$$
\begin{gathered}
D^{Y}=\sum_{i=1}^{2 l} c\left(e_{i}^{\prime}\right) \nabla_{e_{i}^{\prime}}^{B, Y}+2 c\left(B_{Y}\right) \\
D_{T}^{X}=\sum_{i=1}^{2 n} c\left(e_{i}\right) \nabla_{e_{i}}^{B, X}+2 c\left(B_{X}\right)+\sqrt{-1} T(\hat{c}(v)+\hat{c}(\bar{v})) .
\end{gathered}
$$

\section{c) The local behavior of the deformed operator $D_{T}^{X}$ near $Y$}


For $y \in Y$ and $Z \in N_{\mathbf{R}, y}$, let $t \in \mathbf{R} \rightarrow x_{t}=\exp _{y}^{X}(t Z) \in X$ be the geodesic in $X$ with respect to the Levi-Civita connection $\nabla^{L^{X}}$, such that $x_{0}=y, d x /\left.d t\right|_{t=0}=Z$. For $\epsilon>0$, let $B_{\epsilon}=\left\{Z \in N_{\mathbf{R}}|| Z \mid<\epsilon\right\}$. Since $X$ and $Y$ are compact, there exists an $\epsilon_{0}>0$ such that for $0<\epsilon<\epsilon_{0}$, the map $(y, Z) \in N_{\mathbf{R}} \rightarrow \exp _{y}^{X}(Z) \in X$ is a diffeomorphism from $B_{\epsilon}$ to a tubular neighborhood $\mathcal{U}_{\epsilon}$ of $Y$ in $X$. From now on, we will identify $B_{\epsilon}$ with $\mathcal{U}_{\epsilon}$ and use the notation $x=(y, Z)$ instead of $x=\exp _{y}^{X}(Z)$.

We will make use of the trivialization of $\left.\left(\Lambda^{(\cdot)}\left(T_{\mathbf{C}}^{*} X\right) \otimes \xi\right)\right|_{\mathcal{U}_{\epsilon_{0}}}$ by the parallel transport of $\left.\left(\Lambda^{(\cdot)}\left(T_{\mathbf{C}}^{*} X\right) \otimes \xi\right)\right|_{Y}$ with respect to the connection $\nabla^{B, X}$ along the geodesic $t \rightarrow(y, t Z)$. The key point here is that this trivialization preserves the metric and the $\mathbf{Z}$-grading since the Bismut connection is a unitary connection and preserves the complex structure of $T_{\mathbf{R}} X$. By using the trivialization of $\Lambda^{(\cdot)}\left(T_{\mathbf{C}}^{*} X\right) \otimes \xi$ over $\mathcal{U}_{\epsilon_{0}}$, we can and will make the identification of $\left.\left(\Lambda^{(\cdot)}\left(T_{\mathbf{C}}^{*} X\right) \otimes \xi\right)\right|_{\mathcal{U}_{\epsilon_{0}}}$ with $\left.\pi^{*}\left(\left.\left(\Lambda^{(\cdot)}\left(T_{\mathbf{C}}^{*} X\right) \otimes \xi\right)\right|_{Y}\right)\right|_{B_{\epsilon_{0}}}$ and so we can consider $\nabla^{B, X}$ as a unitary connection on the Hermitian vector bundle $\left.\pi^{*}\left(\left.\left(\Lambda^{(\cdot)}\left(T_{\mathbf{C}}^{*} X\right) \otimes \xi\right)\right|_{Y}\right)\right|_{B_{\epsilon_{0}}}$ with the obviously induced metric. Note that there exists another unitary connection $\nabla^{B, X, \oplus}$ on $\pi^{*}\left(\left.\left(\Lambda^{(\cdot)}\left(T_{\mathbf{C}}^{*} X\right) \otimes \xi\right)\right|_{Y}\right)$ defined by

$$
\nabla^{B, X, \oplus}=\pi^{*}\left(\nabla^{-B_{X}, \oplus} \otimes 1+1 \otimes j^{*} \nabla^{\Lambda^{*}\left(\widetilde{T^{*} X}\right) \otimes \xi}\right) .
$$

Let $d v_{Y}$ (resp. $\left.d v_{N}\right)$ denote the Riemannian volume element of $\left(Y, g^{T Y}\right)$ (resp. the fibres of $\left.\left(N, g^{N}\right)\right)$. We define a smooth positive function $k(y, Z)$ on $B_{\epsilon_{0}}$ by the equation $d v_{X}(y, Z)=k(y, Z) d v_{Y}(y) d v_{N_{y}}(Z)$ and an $L^{2}$-Hermitian inner product on $\mathbf{E}$ by

$$
\langle\langle f, g\rangle\rangle=\frac{1}{(2 \pi)^{\operatorname{dim}_{\mathbf{C}} X}} \int_{Y} \int_{N_{\mathbf{R}, y}}\langle f, g\rangle(y, Z) d v_{N}(Z) d v_{Y}(y),
$$

for any $f, g \in \mathbf{E}$ with compact support, where $\mathbf{E}$ denotes the set of smooth sections of $\pi^{*}\left(\left.\left(\Lambda^{(\cdot)}\left(T_{\mathbf{C}}^{*} X\right) \otimes \xi\right)\right|_{Y}\right)$ on $N_{\mathbf{R}}$. Clearly, $k(y)=k(y, 0)=1$ on $Y$ and $k(y, Z)$ has a positive lower bound on $\mathcal{U}_{\epsilon_{0} / 2}$. If $f \in \mathbf{E}$ has compact support in $B_{\epsilon_{0}}$, we can identify $f$ with an element in $\Omega^{(\cdot)}(X, \xi)$ which has compact support in $\mathcal{U}_{\epsilon_{0}}$.

Let $T N_{\mathbf{R}}=T^{H} N_{\mathbf{R}} \oplus N_{\mathbf{R}}$ be the splitting of $T N_{\mathbf{R}}$ induced by the Euclidean connection $\nabla^{L^{N}}=P^{N_{\mathbf{R}}}\left(j^{*} \nabla^{L^{X}}\right) P^{N_{\mathbf{R}}}$ on $N_{\mathbf{R}}$, where $T^{H} N_{\mathbf{R}}$ denotes the horizontal part of $T N_{\mathbf{R}}$. If $U \in T_{\mathbf{R}} Y$, let $U^{H} \in T^{H} N_{\mathbf{R}}$ denote the horizontal lift of $U$ in $T^{H} N_{\mathbf{R}}$, so that $\pi_{*} U^{H}=U$. Let

$$
\left\{e_{1}, \ldots, e_{2 l}, e_{2 l+1}, \ldots, e_{2 n}\right\}
$$

be an orthonormal basis of $\left.T_{\mathbf{R}} X\right|_{Y}$ with $\left\{e_{1}, \ldots, e_{2 l}\right\}$ an orthonormal basis of $T_{\mathbf{R}} Y$ and $\left\{e_{2 l+1}, \ldots, e_{2 n}\right\}$ an orthonormal basis of $N_{\mathbf{R}}$. 
Definition 2.2 Let $D^{H}$, $D^{N}$ be the operators acting on $\mathbf{E}$

$$
D^{H}=\sum_{i=1}^{2 l} c\left(e_{i}\right) \nabla_{e_{i}^{H}}^{B, X, \oplus}+2 c\left(B_{Y}\right), \quad D^{N}=\sum_{\alpha=2 l+1}^{2 n} c\left(e_{\alpha}\right) \nabla_{e_{\alpha}}^{B, X, \oplus} .
$$

Clearly, $D^{N}$ acts along the fibres $N_{\mathbf{R}, y}$ as the operator $\sqrt{2}\left(\bar{\partial}^{N_{y}}+\bar{\partial}^{N_{y} *}\right)$. Note that $D^{H}$, $D^{N}$ are self-adjoint with respect to the Hermitian inner product (2.23).

Now we turn to Taylor expansions of $v$ near $Y$ along the geodesic $(y, t Z)$ for $y \in Y$ and $Z \in N_{\mathbf{R}, y}$. Let $\left\{w_{1}, \ldots, w_{l}, w_{l+1}, \ldots, w_{n}\right\}$ be a unitary basis for $\left.T X\right|_{Y}$ and let $\left(z^{l+1}, \ldots, z^{n}\right)$ denote the associated holomorphic coordinate system on $N_{y}$ with $w_{\alpha}=\sqrt{2} \partial / \partial z^{\alpha}$ for $l+1 \leq \alpha \leq n$. Note that $\mathbf{L}_{v}: N \rightarrow \widetilde{N}$ is unitary fiberwisely. Set

$$
\tilde{w}_{\alpha}=\mathbf{L}_{v}\left(w_{\alpha}\right), \quad \tilde{\bar{w}}_{\alpha}=\mathbf{L}_{v}\left(\bar{w}_{\alpha}\right), \quad l+1 \leq \alpha \leq n .
$$

Hence, $\left\{w_{1}, \ldots, w_{l}, \tilde{w}_{l+1}, \ldots, \tilde{w}_{n}\right\}$ is a unitary basis for $\left.\widetilde{T X}\right|_{Y}$. We use $\tilde{w}^{\tau}$ to denote the parallel transport of $\tilde{w}$ with respect to the holomorphic Hermitian connection $\nabla^{\widetilde{T X}}$ along the geodesic $(y, t Z)$. We write $v$ on $\mathcal{U}_{\epsilon}$ as

$$
v(y, Z)=\frac{1}{\sqrt{2}}\left(\sum_{i=1}^{l} v^{i}(y, Z) w_{i}^{\tau}+\sum_{\alpha=l+1}^{n} v^{\alpha}(y, Z) \tilde{w}_{\alpha}^{\tau}\right)
$$

for some smooth functions $v^{i}$ and $v^{\alpha}$. Set

$$
\begin{gathered}
v_{Y, 1}=\frac{1}{\sqrt{2}} \sum_{i=1}^{l} \sum_{\alpha=l+1}^{n} \frac{\partial v^{i}}{\partial z^{\alpha}}(y) z^{\alpha} w_{i}, v_{N, 1}=\frac{1}{\sqrt{2}} \sum_{\alpha, \beta=l+1}^{n} \frac{\partial v^{\alpha}}{\partial z^{\beta}}(y) z^{\beta} \tilde{w}_{\alpha} \\
v_{Y, 2}=\frac{1}{2 \sqrt{2}} \sum_{i=1}^{l} \sum_{\alpha, \beta=l+1}^{n} \frac{\partial^{2} v^{i}}{\partial z^{\alpha} \partial z^{\beta}}(y) z^{\alpha} z^{\beta} w_{i}, v_{N, 2}=\frac{1}{2 \sqrt{2}} \sum_{\alpha, \beta, \gamma=l+1}^{n} \frac{\partial^{2} v^{\alpha}}{\partial z^{\beta} \partial z^{\gamma}}(y) z^{\beta} z^{\gamma} \tilde{w}_{\alpha} .
\end{gathered}
$$

Since $v$ is transversal, we get by the definition $(2.26)$,

$$
v_{Y, 1}=0, \quad v_{N, 1}=-\frac{1}{\sqrt{2}} \sum_{\alpha=l+1}^{n} z^{\alpha} \tilde{w}_{\alpha}
$$

and so

$$
v(y, Z)=v_{N, 1}(y, Z)+v_{Y, 2}(y, Z)+v_{N, 2}(y, Z)+O\left(|Z|^{3}\right) .
$$


Define

$$
D_{T}^{N}=D^{N}+\sqrt{-1} T\left(\hat{c}\left(v_{N, 1}\right)+\hat{c}\left(\bar{v}_{N, 1}\right)\right) .
$$

A direct and easy computation shows that

$$
\left(D_{T}^{N}\right)^{2}=-4 \sum_{\alpha=l+1}^{n} \frac{\partial^{2}}{\partial z^{\alpha} \partial \bar{z}^{\alpha}}+T^{2}|Z|^{2}-\sqrt{-1} T \sum_{\alpha=l+1}^{n}\left(c\left(\bar{w}_{\alpha}\right) \hat{c}\left(\tilde{w}_{\alpha}\right)+c\left(w_{\alpha}\right) \hat{c}\left(\overline{\tilde{w}}_{\alpha}\right)\right) .
$$

Set

$$
\theta=\sum_{\alpha=l+1}^{n} w_{\alpha}^{*} \wedge \overline{\tilde{w}}_{\alpha}^{*}
$$

Clearly, $\theta$ is a well-defined smooth section of $\Lambda^{(\cdot)}\left(\bar{N}^{*} \otimes \widetilde{N}^{*}\right)$ over $Y$ of the degree 0 . Now we have the following analogue of [BL, Proposition 7.3]:

Lemma 2.3 Take $T>0$. Then for any $y \in Y$, the operator $\left(D_{T}^{N}\right)^{2}$ acting on $\Gamma\left(\pi^{*} \Lambda^{(\cdot)}\left(\bar{N}_{y}^{*} \oplus \widetilde{N}_{y}\right)\right)$ over $N_{y}$ is nonnegative with the kernel $\mathbf{C}\left\{\beta_{y}\right\}$, where

$$
\beta_{y}=\exp \left(\theta_{y}-\frac{T}{2}|Z|^{2}\right), \quad\left|\exp \theta_{y}\right|_{\Lambda^{(\cdot)}\left(\bar{N}_{y}^{*} \oplus \widetilde{N}_{y}\right)}=2^{(n-l) / 2} .
$$

Moreover, the nonzero eigenvalues of $\left(D_{T}^{N}\right)^{2}$ are all $\geq T A$ for some positive constant $A$ which can be chosen to be independent of $y$.

Proof. The proof of the lemma is standard (cf. [BL, Sect.7, (7.10)-(7.13)]; also cf. [Z1, Chapter 4, Sect. 4.5]).

For any $y \in Y, Z \in N_{\mathbf{R}, y}$, let $\tau U$ denote the parallel transport of $U \in T_{\mathbf{R}, y} X$ with respect to the Levi-Civita connection $\nabla^{L^{X}}$ along the geodesic $(y, t Z)$. Note that we have identified the bundle $\left.\pi^{*}\left(\left.\left(\Lambda^{(\cdot)}\left(T_{\mathbf{C}}^{*} X\right) \otimes \xi\right)\right|_{Y}\right)\right|_{B_{\epsilon_{0}}}$ with the bundle $\left.\left(\Lambda^{(\cdot)}\left(T_{\mathbf{C}}^{*} X\right) \otimes \xi\right)\right|_{\mathcal{U}_{0}}$ by trivializing the later bundle along the geodesic $(y, t Z)$ by using the connection $\nabla^{B, X}$. The Clifford action of $c((\tau U)(y, t Z))$ on $\Lambda^{(\cdot)}\left(T_{\mathbf{C}}^{*} X\right) \otimes \xi$ is generally not constant along the geodesic $(y, t Z)$. This is different from the situation in [BL, Sect.8], where the connection on the related bundle is the lifting of the Levi-Civita connection on $T X$ since the manifold $X$ is Kähler. Hence, to obtain an analogue of [BL, Theorem 8.18], we need to work out the difference between $c((\tau U)(y, Z))$ and the constant Clifford action $c(U)$ on $\pi^{*}\left(\left(\Lambda^{(\cdot)}\left(T_{\mathbf{C}}^{*} X\right) \otimes\right.\right.$ $\left.\xi)\left.\right|_{Y}\right)\left.\right|_{B_{\epsilon_{0}}}$. Since $\nabla^{-B_{X}}=\nabla^{L^{X}}+S^{-B_{X}}$ is unitary, we know that

$$
\left[\nabla_{Z}^{B, X}, c((\tau U)(y, t Z))\right]_{\left.\right|_{t=0}}=c\left(\nabla_{Z}^{-B_{X}}(\tau U)(y, t Z)\right)_{\mid t=0}=c\left(S^{-B_{X}}(Z) U\right),
$$


thus

$$
c((\tau U)(y, Z))=c(U)+c\left(S^{-B_{X}}(Z) U\right)+O\left(|Z|^{2}\right) .
$$

Set with respect to the basis $(2.24)$ :

$$
\begin{gathered}
M=\frac{1}{2} \sum_{i, j}^{2 l} \sum_{\alpha=2 l+1}^{2 n}\left\langle\mathbf{A}\left(e_{i}\right) e_{j}, e_{\alpha}\right\rangle c\left(e_{i}\right) c\left(e_{j}\right) c\left(e_{\alpha}\right)-\frac{1}{2} \sum_{\alpha=2 l+1}^{2 n}\left(e_{\alpha} k\right) c\left(e_{\alpha}\right) \\
c\left(B^{\prime}(y)\right)=\frac{1}{2} \sum_{i, j=1}^{2 l} \sum_{\alpha=2 l+1}^{2 n} B_{X}\left(e_{i}, e_{j}, e_{\alpha}\right) c\left(e_{i}\right) c\left(e_{j}\right) c\left(e_{\alpha}\right) \\
+\frac{1}{6} \sum_{\alpha, \beta, \gamma=2 l+1}^{2 n} B_{X}\left(e_{\alpha}, e_{\beta}, e_{\gamma}\right) c\left(e_{\alpha}\right) c\left(e_{\beta}\right) c\left(e_{\gamma}\right) \\
c\left(B^{\prime \prime}(y)\right)=\frac{1}{2} \sum_{i=1}^{2 l} \sum_{\alpha, \beta=2 l+1}^{2 n} B_{X}\left(e_{i}, e_{\alpha}, e_{\beta}\right) c\left(e_{i}\right) c\left(e_{\alpha}\right) c\left(e_{\beta}\right)
\end{gathered}
$$

One verifies easily that

$$
c\left(B_{X}(y)\right)=c\left(B_{Y}(y)\right)+c\left(B^{\prime}(y)\right)+c\left(B^{\prime \prime}(y)\right) .
$$

Now we have the following analogue of [BL, Theorem 8.18], which describes the local behavior of $D_{T}^{X}$ near $Y$. Comparing to [BL, Theorem 8.18, (8.58)], some new terms enter into the following theorem.

Theorem 2.4 As $T \rightarrow+\infty$, then

$$
k^{1 / 2} D_{T}^{X} k^{-1 / 2}=D^{H}+D_{T}^{N}+M_{T}+c\left(B^{\prime \prime}\right)+T \sqrt{-1} \hat{c}\left(v_{Y, 2}+\bar{v}_{Y, 2}\right)+\mathbf{S}+R_{T},
$$

where

$$
\begin{gathered}
M_{T}=M+c\left(B^{\prime}\right)+T \sqrt{-1} \hat{c}\left(v_{N, 2}+\bar{v}_{N, 2}\right), \\
\mathbf{S}=-\sum_{i=1}^{2 n} c\left(e_{i}\right) \nabla_{P^{N} \mathbf{R} S^{-B} X}^{B, X, \oplus}(Z) e_{i} \\
R_{T}=O\left(|Z| \partial^{H}+|Z|^{2} \partial^{N}+|Z|+T|Z|^{3}\right),
\end{gathered}
$$

and $\partial^{H}, \partial^{N}$ represent horizontal and vertical differential operators, respectively. 
Proof. Let $\left\{\tau e_{1}, \ldots, \tau e_{2 n}\right\}$ be the parallel transport of the basis (2.24) with respect to the Levi-Civita connection $\nabla^{L^{X}}$ along the geodesic $(y, t Z)$ for $y \in Y$ and $Z \in N_{\mathbf{R}, y}$. From (2.21), we have

$$
D_{T}^{X}=\sum_{i=1}^{2 n} c\left(\tau e_{i}\right) \nabla_{\tau e_{i}}^{B, X}+2 c\left(B_{X}\right)+\sqrt{-1} T(\hat{c}(v)+\hat{c}(\bar{v})) .
$$

We identify $\overline{T X}$ with $\pi^{*}\left(\left.\overline{T X}\right|_{Y}\right)$ over $\mathcal{U}_{\epsilon_{0}}$ by trivializing $\overline{T X}$ with respect to the Bismut connection $\nabla^{-B_{X}}$ along the geodesic $(y, t Z)$ and set

$$
\Gamma=\nabla^{-B_{X}}-\nabla^{-B_{X}, \oplus} .
$$

Let $\Gamma^{\wedge}$ denote the lifting action of $\Gamma$ on $\pi^{*}\left(\left.\Lambda^{\cdot}\left(\overline{T^{*} X}\right)\right|_{Y}\right)$. For any $y \in Y$, we find by $(2.12)$

$$
\sum_{i=1}^{2 n} c\left(e_{i}\right) \Gamma_{y}^{\wedge}\left(e_{i}\right)=\frac{1}{2} \sum_{i, j}^{2 l} \sum_{\alpha=2 l+1}^{2 n}\left\langle\mathbf{A}_{y}\left(e_{i}\right) e_{j}, e_{\alpha}\right\rangle c\left(e_{i}\right) c\left(e_{j}\right) c\left(e_{\alpha}\right) .
$$

Furthermore, recall (2.13) and then we get

$$
\begin{aligned}
k^{1 / 2} D_{T}^{X} k^{-1 / 2}= & \sum_{i=1}^{2 n} c\left(\tau e_{i}\right) \nabla_{\tau e_{i}}^{B, X, \oplus}+2 c\left(B_{X}\right)+\sqrt{-1} T(\hat{c}(v)+\hat{c}(\bar{v})) \\
& +\sum_{i=1}^{2 n} c\left(e_{i}\right) \Gamma_{y}^{\wedge}\left(e_{i}\right)-\frac{1}{2} \sum_{\alpha=2 l+1}^{2 n}\left(e_{\alpha} k\right)(y) c\left(e_{\alpha}\right)+O(|Z|) .
\end{aligned}
$$

Note that (2.31), (2.38), (2.41), (2.43) and (2.48), we have

$$
\begin{aligned}
k^{1 / 2} D_{T}^{X} k^{-1 / 2}= & \sum_{i=1}^{2 n} c\left(\tau e_{i}\right) \nabla_{\tau e_{i}}^{B, X, \oplus}+2 c\left(B_{Y}\right)+T \sqrt{-1} \hat{c}\left(v_{N, 1}+\bar{v}_{N, 1}\right) \\
& +M_{T}+c\left(B^{\prime \prime}\right)+T \sqrt{-1} \hat{c}\left(v_{Y, 2}+\bar{v}_{Y, 2}\right)+O\left(|Z|+T|Z|^{3}\right) .
\end{aligned}
$$

By (2.37) and the expansion of $\tau e_{i}$ along $(y, t Z)$ in the proof of [BL, Theorem 8.18], especially [BL, (8.80), (8.84)], we have

$$
\begin{aligned}
\sum_{i=1}^{2 n} c\left(\tau e_{i}\right) \nabla_{\tau e_{i}}^{B, X, \oplus}= & \sum_{i=1}^{2 l} c\left(e_{i}\right) \nabla_{e_{i}^{H}}^{B, X, \oplus}+\sum_{\alpha=2 l+1}^{2 n} c\left(e_{\alpha}\right) \nabla_{e_{\alpha}}^{B, X, \oplus} \\
& +\sum_{i=1}^{2 n} c\left(S^{-B_{X}}(Z) e_{i}\right) \nabla_{e_{i}}^{B, X, \oplus}+O\left(|Z| \partial^{H}+|Z|^{2} \partial^{N}\right),
\end{aligned}
$$


and then by the definition of $D^{H}$ and $D_{T}^{N}$,

$$
\begin{aligned}
k^{1 / 2} D_{T}^{X} k^{-1 / 2}= & D^{H}+D_{T}^{N}+M_{T}+c\left(B^{\prime \prime}\right)+T \sqrt{-1} \hat{c}\left(v_{Y, 2}+\bar{v}_{Y, 2}\right) \\
& +\sum_{i=1}^{2 n} c\left(S^{-B_{X}}(Z) e_{i}\right) \nabla_{e_{i}}^{B, X, \oplus}+O\left(|Z| \partial^{H}+|Z|^{2} \partial^{N}+|Z|+T|Z|^{3}\right) .
\end{aligned}
$$

But

$$
\begin{aligned}
\sum_{i=1}^{2 n} c\left(S^{-B_{X}}(Z) e_{i}\right) \nabla_{e_{i}}^{B, X, \oplus} & =-\sum_{i=1}^{2 n} c\left(e_{i}\right) \nabla_{S^{-B} X(Z) e_{i}}^{B, X, \oplus} \\
& =-\sum_{i=1}^{2 n} c\left(e_{i}\right) \nabla_{P^{N} \mathbf{R}^{-B_{X}}(Z) e_{i}}^{B, X, \oplus}-\sum_{i=1}^{2 n} c\left(e_{i}\right) \nabla_{P^{T} \mathbf{R}^{Y} S^{-B_{X}}(Z) e_{i}}^{B, X, \oplus} \\
& =\mathbf{S}+O\left(|Z| \partial^{H}\right),
\end{aligned}
$$

from which we complete the proof of the theorem.

\section{The proof of Theorem 1.1}

In this section, we prove Theorem 1.1 by using Bismut-Lebeau's techniques in [BL, Sect.9, Sect.10, a)].

For any $\mu \geq 0$, let $\mathrm{E}^{\mu}$ (resp. $\mathbf{E}^{\mu}$, resp. $\mathrm{F}^{\mu}$ ) be the set of sections of $\Lambda^{(\cdot)}\left(T_{\mathbf{C}}^{*} X\right) \otimes \xi$ on $X$ (resp. of $\pi^{*}\left(\left.\left(\Lambda^{(\cdot)}\left(T_{\mathbf{C}}^{*} X\right) \otimes \xi\right)\right|_{Y}\right)$ on the total space of $N$, resp. of $\left.\Lambda^{(\cdot)}\left(T_{\mathbf{C}}^{*} Y\right) \otimes \xi\right|_{Y}$ on $\mathrm{Y}$ ) which lie in the $\mu$-th Sobolev spaces. Let \|\|$_{\mathrm{E}^{\mu}}$ (resp. \|\|$_{\mathrm{E}^{\mu}}$, resp. \|\|$_{\mathrm{F}^{\mu}}$ ) be the Sobolev norm on $\mathrm{E}^{\mu}$ (resp. $\mathbf{E}^{\mu}$, resp. $\mathrm{F}^{\mu}$ ). We always assume that the norms $\|\quad\|_{E^{0}}$ (resp. \|\|$_{\mathbf{E}^{0}}$, resp. \|\|$_{\mathrm{F}^{0}}$ is the norm associated with the scalar products on the corresponding bundles).

Let $\gamma: \mathbf{R} \rightarrow[0,1]$ be a smooth even function with $\gamma(a)=1$ if $|a| \leq \frac{1}{2}$ and $\gamma(a)=0$ if $|a| \geq 1$. For any $y \in Y, Z \in N_{y}$ and $\epsilon \in\left(0, \epsilon_{0}\right)$, where $\epsilon_{0}$ is chosen as in Section 2, c), set

$$
\gamma_{\epsilon}(Z)=\gamma\left(\frac{|Z|}{\epsilon}\right), \quad \alpha_{T}=\int_{N_{\mathbf{R}, y}} \gamma_{\epsilon}^{2}(Z) \exp \left(-T|Z|^{2}\right) \frac{d v_{N_{y}}(Z)}{(2 \pi)^{\operatorname{dim}_{\mathbf{C}} N}} .
$$

Clearly, $\alpha_{T}$ does not depend on $y \in Y$ and $\alpha_{T}=O\left(\frac{1}{T^{n-l}}\right)$.

For $\mu \geq 0, T>0$, define linear maps $I_{T}: \mathrm{F}^{\mu} \rightarrow \mathbf{E}^{\mu}$ and $J_{T}: \mathrm{F}^{\mu} \rightarrow \mathrm{E}^{\mu}$ by

$$
I_{T} u=\left(\frac{1}{2^{n-l} \alpha_{T}}\right)^{1 / 2} \gamma_{\epsilon}(Z)\left(\pi^{*} u\right) \beta_{y}, J_{T} u=k^{-1 / 2} I_{T} u, \quad \forall u \in \mathrm{F}^{\mu} .
$$


It is easy to see that $I_{T}, J_{T}$ are isometries from $\mathrm{F}^{0}$ onto their images. For $\mu \geq 0, T>0$, let $\mathbf{E}_{T}^{\mu}\left(\right.$ resp. $\left.\mathrm{E}_{T}^{\mu}\right)$ be the image of $\mathrm{F}^{\mu}$ in $\mathbf{E}^{\mu}\left(\right.$ resp. $\left.\mathrm{E}^{\mu}\right)$ under $I_{T}\left(\operatorname{resp} . J_{T}\right)$ and let $\mathbf{E}_{T}^{0, \perp}$ (resp. $\mathrm{E}_{T}^{0, \perp}$ ) be the orthogonal complement of $\mathbf{E}_{T}^{0}$ (resp. $\mathrm{E}_{T}^{0}$ ) in $\mathbf{E}^{0}$ (resp. $\mathrm{E}^{0}$ ) and let $p_{T}$, $p_{T}^{\perp}$ (resp. $\bar{p}_{T}, \bar{p}_{T}^{\perp}$ ) be the orthogonal projection operators from $\mathbf{E}^{0}$ (resp. $\mathrm{E}^{0}$ ) onto $\mathbf{E}_{T}^{0}, \mathbf{E}_{T}^{0, \perp}$ (resp. $\left.\mathrm{E}_{T}^{0}, \mathrm{E}_{T}^{0, \perp}\right)$, respectively. Set

$$
\mathrm{E}^{\mu, \perp}=\mathrm{E}^{\mu} \cap \mathrm{E}_{T}^{0, \perp}
$$

Then $\mathrm{E}^{0}$ splits orthogonally into

$$
\mathrm{E}^{0}=\mathrm{E}_{T}^{0} \oplus \mathrm{E}_{T}^{0, \perp}
$$

Since the map $s \in \mathbf{E}^{0} \rightarrow k^{-1 / 2} s \in \mathrm{E}^{0}$ is an isometry, we see that the map $s \rightarrow k^{-1 / 2} s$ identifies the Hilbert space $\mathbf{E}_{T}^{0}$ and $\mathrm{E}_{T}^{0}$. Corresponding to the decomposition (3.4) we set:

$$
D_{T, 1}=\bar{p}_{T} D_{T}^{X} \bar{p}_{T}, \quad D_{T, 2}=\bar{p}_{T} D_{T}^{X} \bar{p}_{T}^{\perp}, \quad D_{T, 3}=\bar{p}_{T}^{\perp} D_{T}^{X} \bar{p}_{T}, \quad D_{T, 4}=\bar{p}_{T}^{\perp} D_{T}^{X} \bar{p}_{T}^{\perp} .
$$

Then

$$
D_{T}^{X}=D_{T, 1}+D_{T, 2}+D_{T, 3}+D_{T, 4}
$$

We have the following analogue of [BL, Theorem 9.8].

Lemma 3.1 The following formula holds on $\Gamma\left(\left.\Lambda^{(\cdot)}\left(T_{\mathbf{C}}^{*} Y\right) \otimes \xi\right|_{Y}\right)$ as $T \rightarrow+\infty$

$$
J_{T}^{-1} D_{T, 1} J_{T}=D^{Y}+O\left(\frac{1}{\sqrt{T}}\right)
$$

where $O\left(\frac{1}{\sqrt{T}}\right)$ is a first order differential operator with smooth coefficients dominated by $C / \sqrt{T}$.

Proof. Note that the action of the operator $M_{T}$ on $\pi^{*}\left(\left.\left(\Lambda^{(\cdot)}\left(T_{\mathbf{C}}^{*} X\right) \otimes \xi\right)\right|_{Y}\right)$ interchanges $\pi^{*}\left(\Lambda^{\text {(even) }}\left(\bar{N}^{*} \oplus \widetilde{N}^{*}\right)\right)$ and $\pi^{*}\left(\Lambda^{(\text {odd })}\left(\bar{N}^{*} \oplus \widetilde{N}^{*}\right)\right)$, we get

$$
p_{T} M_{T} p_{T}=0
$$

Note that $B_{X}$ is antisymmetric and $\left\langle c\left(e_{\alpha}\right) c\left(e_{\beta}\right) \beta_{y}, \beta_{y}\right\rangle=0$ for any $\alpha, \beta$ with $2 l+1 \leq \alpha<$ $\beta \leq 2 n$, we obtain

$$
p_{T} c\left(B^{\prime \prime}\right) p_{T}=0
$$


Since $B_{X}$ is antisymmetric, by $(2.8)$ we get $\left\langle S^{-B_{X}}(Z) e_{i}, Z\right\rangle=0$ and so

$$
\nabla_{P^{N} \mathbf{R} S^{-B_{X}}(Z) e_{i}}^{B, X, \oplus}\left(\gamma_{\epsilon}(Z) \exp \left(\theta_{y}-\frac{T|Z|^{2}}{2}\right)\right)=\exp \left(\theta_{y}-\frac{T|Z|^{2}}{2}\right)\left(P^{N_{\mathbf{R}}} S^{-B_{X}}(Z) e_{i}\right) \gamma_{\epsilon}(Z)
$$

From the equality above we can prove easily the following estimate for some uniformly positive constant $C$ and any $s \in \mathbf{E}^{1}$ :

$$
\left\|p_{T} \mathbf{S} s\right\|_{\mathbf{E}^{0}} \leq \frac{C}{\sqrt{T}}\|s\|_{\mathbf{E}^{1}}
$$

Since $\int_{\mathbf{C}} e^{-T|z|^{2}} z^{2} d z d \bar{z}=0$ and $\left(\gamma_{\epsilon}^{2}-1\right)$ vanishes on a symmetric domain containing 0 , we have for any $u \in \mathrm{F}$ that

$$
I_{T}^{-1} p_{T}\left(\hat{c}\left(v_{Y, 2}\right)+\hat{c}\left(\bar{v}_{Y, 2}\right)\right)\left(\frac{1}{2^{n-l} \alpha_{T}}\right)^{1 / 2} \gamma_{\epsilon}(Z)\left(\pi^{*} u\right) \beta_{y}=O\left(\frac{1}{T^{3 / 2}}\right) .
$$

On the other hand, note that $\beta_{y}$ is of constant length on $Y$, we get for $1 \leq i \leq 2 l$ that

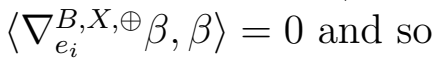

$$
I_{T}^{-1} p_{T} D^{H} p_{T} I_{T}=D^{Y}
$$

One can then proceed as in [BL, Proof of Theorem 9.8] and use (3.8)-(3.12) to complete the proof of Lemma 3.1 easily.

Note that the estimate (3.12) and proceed as the proof of Theorem 9.10, Theorem 9.11 and Theorem 9.14 in [BL, Sect.9], one can prove the following lemma without any new difficulty.

Lemma 3.2 There exist $C_{1}>0, C_{2}>0$ and $T_{0}>0$ such that for any $T \geq T_{0}$, $s \in \mathrm{E}_{T}^{1, \perp}$ and $s^{\prime} \in \mathrm{E}_{T}^{1}$, then

$$
\begin{gathered}
\left\|D_{T, 2} s\right\|_{\mathrm{E}^{0}} \leq C_{1}\left(\frac{\|s\|_{\mathrm{E}^{1}}}{\sqrt{T}}+\|s\|_{\mathrm{E}^{0}}\right), \\
\left\|D_{T, 3} s^{\prime}\right\|_{\mathrm{E}^{0}} \leq C_{1}\left(\frac{\left\|s^{\prime}\right\|_{\mathrm{E}^{1}}}{\sqrt{T}}+\left\|s^{\prime}\right\|_{\mathrm{E}^{0}}\right), \\
\left\|D_{T, 4} s\right\|_{\mathrm{E}^{0}} \geq C_{2}\left(\|s\|_{\mathrm{E}^{1}}+\sqrt{T}\|s\|_{\mathrm{E}^{0}}\right) .
\end{gathered}
$$


Let Spec $\left(D^{Y}\right)$ denote the spectrum of $D^{Y}$. Choose $c>0$ such that $\operatorname{Spec}\left(D^{Y}\right) \cap$ $[-2 c, 2 c] \subset\{0\}$. Let $\delta=\{\lambda \in \mathbf{C}:|\lambda|=c\}$. Let $\mathrm{E}_{c}(T)$ denote the direct sum of the eigenspaces of $D_{T}^{X}$ with eigenvalues lying in $[-c, c]$. Then $\mathrm{E}_{c}(T)$ is a finite dimensional subspace of $\mathrm{E}^{0}$. Let $P_{T, c}$ denote the orthogonal projection from $J_{T}\left(\operatorname{ker}\left(D^{Y}\right)\right)$ to $\mathrm{E}_{c}(T)$. By Lemma 3.1 and Lemma 3.2, we have the following analogue of [BL, (9.156)] (also see [TZ, Proposition 4.4] for a proof without using the norm in [BL, Sect.9, Definition 9.17] and the distance in [BL, Sect.9, Definition 9.22]):

Theorem 3.3 There exist $c>0$ and $T_{0}>0$ such that for any $T \geq T_{0}$, the projection

$$
P_{T, c}: J_{T}\left(\operatorname{ker}\left(D^{Y}\right)\right) \rightarrow \mathrm{E}_{c}(T)
$$

is an isomorphism.

Now to prove Theorem 1.1 we only need to prove that when $T$ large enough, $D_{T}^{X}$ has no nonzero small eigenvalues or equivalently, to prove the following equality:

$$
E_{c}(T)=\operatorname{ker}\left(D_{T}^{X}\right)
$$

Let $Q$ denote the orthogonal projection from $\Omega^{(\cdot)}(Y, \xi)$ to $\operatorname{ker}\left(D^{Y}\right)$. Then we have the following analogue of [Z2, Theorem 1.10] (also see [BL, Theorem 10.1, (10.4)]):

Theorem 3.4 There exist $c>0, C>0, T_{1}>0$ such that for any $T \geq T_{1}$, any $\sigma \in \operatorname{ker}\left(D^{Y}\right)$,

$$
\left\|\left(2^{n-l} \alpha_{T}\right)^{1 / 2} Q j^{*} P_{T, c} J_{T} \sigma-\sigma\right\|_{0} \leq \frac{C}{\sqrt{T}}\|\sigma\|_{0}
$$

Proof. The proof of [Z2, Theorem 1.10], which is a modified version of the proof of [BL, Theorem 10.1, (10.4)], is carried out here with the identity [Z2,(1.34)] in the proof of the [Z2, Theorem 1.10] replaced by the following equality

$$
j^{*} \frac{1}{2 \pi \sqrt{-1}} \int_{\delta} k^{-1 / 2} \gamma_{\epsilon} \frac{\left(\pi^{*} \sigma\right) \beta_{y}}{\lambda} d \lambda=\sigma
$$

The proof of (3.20) is similar to that of the identity [BL, (10.29)].

Note that $j^{*} \beta_{y}=1$ is crucial in the proof of (3.19). It is no longer true for the case of the analytic proof of Morse inequalities of Witten ([W]) since in that case the contribution 
of the bundle $\Lambda\left(N^{*}\right)$ to the kernel of $D_{T}^{N}$ is a pure $p$-form around each critical point of index $p$ (cf. [Z1, Chapter 5,6]) and its pull-back by $j^{*}$ vanishes on $Y$. Consequently, $j^{*}$ can not be a quasi-isomorphism at all in that case.

Proof of Theorem 1.1. The proof of Theorem 1.1 now is similar to that in [Z2, Sect.1, e)]. Note that the trick used in Zhang's proof and so ours is inspired by Braverman ([Br, Sect.3]). First of all, we know from Theorem 3.3 that

$$
P_{T, c} J_{T}: \operatorname{ker}\left(D^{Y}\right) \rightarrow \mathrm{E}_{c}(T)
$$

is an isomorphism when $T$ is very large. Take $\alpha \in \mathrm{E}_{c}(T)$. Then $\bar{\partial}_{T}^{X} \alpha \in \mathrm{E}_{c}(T)$. By the above discussion, there exists $\sigma \in \operatorname{ker}\left(D^{Y}\right)$ such that

$$
\bar{\partial}_{T}^{X} \alpha=\left(2^{n-l} \alpha_{T}\right)^{1 / 2} P_{T, c} J_{T} \sigma .
$$

From (3.22) and that $j^{*}$ is a quasi-homomorphism, i.e. $j^{*} \bar{\partial}_{T}^{X}=\bar{\partial}^{Y} j^{*}$, we have

$$
\left(2^{n-l} \alpha_{T}\right)^{1 / 2} Q j^{*} P_{T, c} J_{T} \sigma=Q j^{*} \bar{\partial}_{T}^{X} \alpha=Q \bar{\partial}^{Y} j^{*} \alpha=0 .
$$

From (3.23) and (3.19), we get

$$
\|\sigma\|_{0} \leq \frac{C}{\sqrt{T}}\|\sigma\|_{0},
$$

and so $\sigma=0$ as $T$ large enough. Thus, when $T$ is large enough, we have that

$$
\left.\bar{\partial}_{T}^{X}\right|_{\mathrm{E}_{c}(T)}=0 .
$$

From (3.25) and Theorem 3.3, we have that when $T$ is large enough,

$$
\operatorname{dim} \operatorname{ker}\left(D_{T}^{X}\right)=\operatorname{dim} \mathrm{E}_{c}(T)=\operatorname{dim} \operatorname{ker}\left(D^{Y}\right) .
$$

Now by Theorem 3.4,

$$
j^{*}: \operatorname{ker}\left(D_{T}^{X}\right) \rightarrow \operatorname{ker}\left(D^{Y}\right)
$$

is clearly an injective and so an isomorphism from (3.26).

Acknowledgements The author would like to thank Professors Jean-Michel Bismut and Xiaonan Ma for many helpful discussions from which the author benefits a lot. This work was done while the author was visiting the Institut des Hautes Études Scientifiques in 
Bures-Sur-Yvette. He would like to thank Professor Jean-Pierre Bourguignon and the IHES for their hospitality and support.

\section{References}

[Bis1] J. -M. Bismut, Holomorphic and de Rham torsions, preprint.

[Bis2] J. -M. Bismut, A local index theorem for non Kähler manifolds, Math. Ann. 284(1989), 681-699.

[BL] J. -M. Bismut and G. Lebeau, Complex immersions and Quillen metrics, Publ. Math. IHES. V.74 (1991).

[Bot] R. Bott, A residue formula for holomorphic vector fields, J. Diff. Geom. 1(1967), 311-330.

[Br] M. Braverman, Cohomology of the Mumford quotient, Progress in Math. 198(2001), 47-59.

[CL] J. B. Carrell and D. I. Lieberman, Holomorphic vector fields and Kaehler manifolds, Invent. Math. 21 (1973), 303-309.

[L] K. Liu, Holomorphic equivariant cohomolgy, Math. Ann. 303(1995), 125-148.

[TZ] Y. Tian and W. Zhang, Quantization formula for symplectic manifolds with boundary, Geom. Funct. Anal. 9(1999), 596-640.

[W] E. Witten, Supersymmetry and Morse theory, J. Diff. Geom. 17(1982), 661-692.

[Z1] W. Zhang, Lectures on Chern-Weil Theory and Witten Deformations, Nankai Tracts in Mathematics, V.4, World Scientific, Singapore, 2001.

[Z2] W. Zhang, A holomorphic quantization formula in singular reduction, Commun. Contemp. Math. 1(1999), No.3, 281-293.

Feng: College of Mathematical Sciences, Nankai University, Tianjin, 300071, China; fht@nankai.edu.cn

Current address:

Feng: Institut Des Hautes Études Scientifiques, Bures-Sur-Yvette, 91440, France; feng@ihes.fr 